\title{
Dumbbell-Shaped Rhabdomyosarcoma: Two-Stage Surgical Resection
}

\author{
Mahmoud ElFiky ${ }^{1}$ \\ ${ }^{1}$ Department of Pediatric Surgery, Cairo University, Cairo, Egypt \\ Eur J Pediatr Surg Rep 2015;3:30-32.
}

Address for correspondence Mahmoud ElFiky, MD, Department of Pediatric Surgery, Cairo University, Cairo University Pediatric Hospital, 1 Ali Ibrahim St., Cairo 11431, Egypt (e-mail: mfiky@kasralainy.edu.eg).
Abstract
Keywords
- Rhabdomyosarcoma
- Neonate
- Tumor
- Surgery

Female neonate admitted to our hospital with an abdominal mass and a thigh mass that were connected as a single dumbbell-shaped mass. CT was done on admission that showed cystic swelling in the thigh with intra-abdominal extension passing under inguinal ligament, most probably lymphangioma.

The patient was assessed and prepared for surgery starting with the abdominal part then after two days for the thigh mass. Distal pulses in the lower limbs were assessed intra- and postoperatively following both surgeries. The pathology report showed rhabdomyosarcoma of the embryonal type.

\section{Introduction}

A female neonate aged 28 days was admitted to our hospital by huge swelling in the thigh.

Antenatal history: a full-term neonate with 37-week gestational age was delivered through cesarean section because of cystic swelling in the thigh, in an otherwise uncomplicated pregnancy or delivery. The neonate was the first with positive consanguinity between parents.

On admission, clinically, the patient had a history of a rapidly enlarging tumor in abdomen and left thigh. On examination, the abdomen was severely distended with visible abdominal veins and abdominal mass measuring $65 \times 60 \mathrm{~mm}$. The neonate also had a left thigh mass measuring $85 \times 50 \mathrm{~mm}$ which seemed impending to rupture. The two masses were connected as a single dumbbell-shaped mass with heterogeneous firm consistency.

Routine laboratory tests were done which were normal except urine analysis which showed high power field cells $>100$ and serum $\alpha$-fetoprotein $=760 \mathrm{ng} / \mathrm{mL}$. Computed tomography done on admission showed cystic swelling in the thigh with intra-abdominal extension passing under inguinal ligament, most probably lymphangioma in origin (-Fig. 1). Bilateral ureteric compression and back pressure changes were also evident.

The patient was assessed by pediatric surgeons and practitioners in the pediatric oncology department, and debulking of

received

February 21, 2014

accepted after revision

April 2, 2014

published online

July 17, 2014 the tumor with subsequent chemotherapy was diagnosed, given the aggressive progression of the disease and increased abdominal tension signs which refuted the possibility of a benign lesion. Subsequently, the patient was prepared for surgery ( - Fig. 2). The abdominal part was tackled first. The mass was overridden by the common iliac vessels. It was found to have solid components, which were very necrotic. Complete excision of the mass was done with sparing of the ureters bilaterally and ligation of feeding vessels to the tumor. Intraoperative duplex was done and all distal pulses in both legs were recorded. The intraoperative blood loss was $80 \mathrm{~cm}$, and the anesthesia team requested to abort the procedure after excision of the abdominal part. Patient was transferred to surgical neonatal intensive care unit intubated.

After blood transfusion and resuscitation, the patient was extubated the same day. The abdominal drain had $50 \mathrm{~mL}$ of serosanguineous fluid, and bilaterally, lower limb pulses were maintained at a warm temperature.

Two days later, the neonate was scheduled for operation of the thigh mass. No intraoperative complications were observed. Distal lower limb pulses were intact evidenced by duplex all through the surgery. The mass seemed to arise in medial compartment of the thigh and was excised completely. Postoperatively, the patient was vitally stable and wound drain was removed. The patient was referred to oncology hospital.
License terms Stuttgart · New York

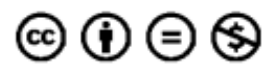




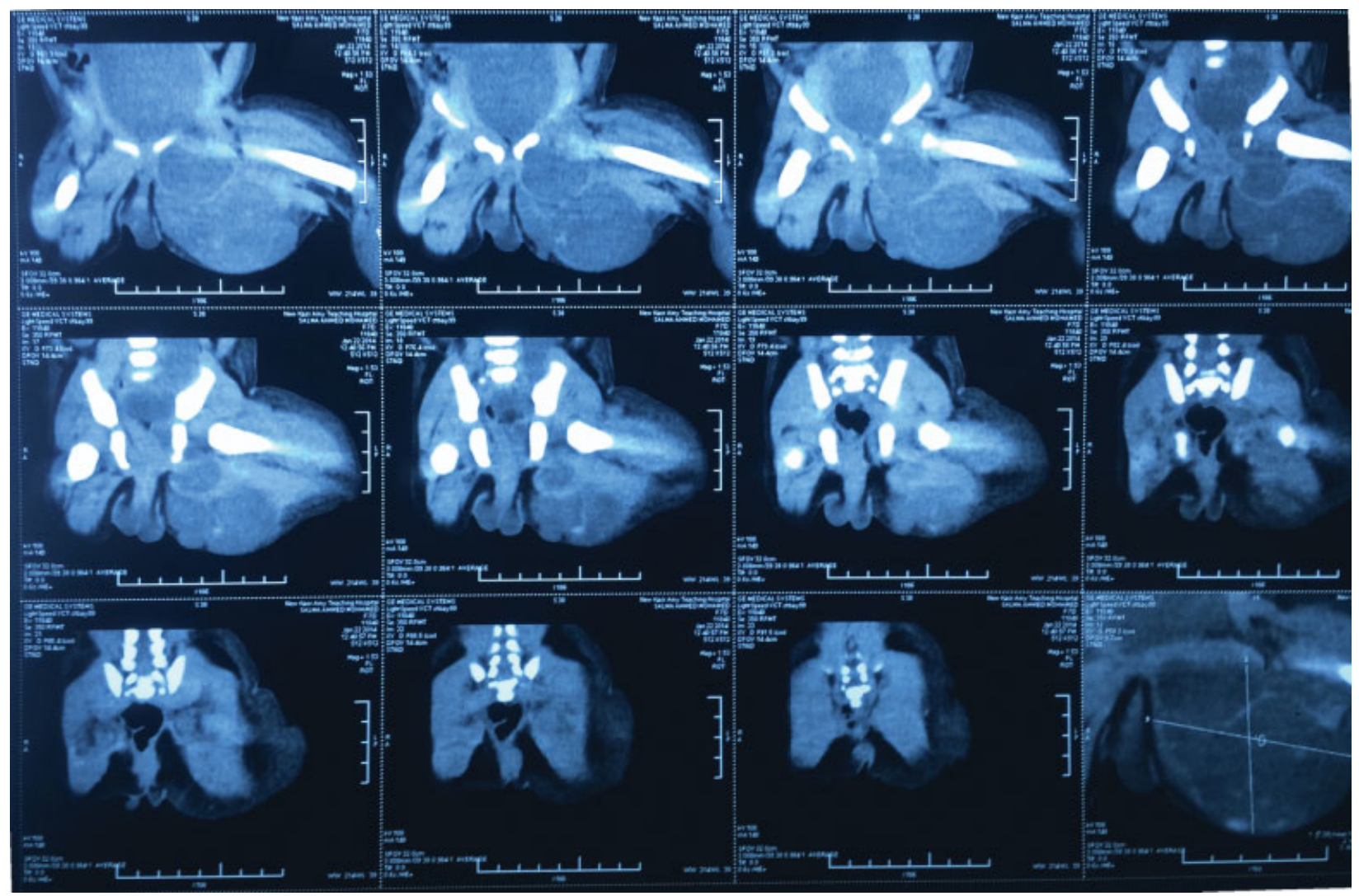

Fig.1 Computed tomography of abdomen and pelvis showing connected masses.

The pathology report showed rhabdomyosarcoma (RMS) of the embryonal type.

\section{Discussion}

\section{Incidence}

Neonatal RMS is a rare tumor (0.4-1\% of RMS). ${ }^{1}$ Nearly, $50 \%$ of tumors occurring in neonates are observed at birth; another 20 to $29 \%$ of tumors become evident within the first week of life. ${ }^{2,3}$

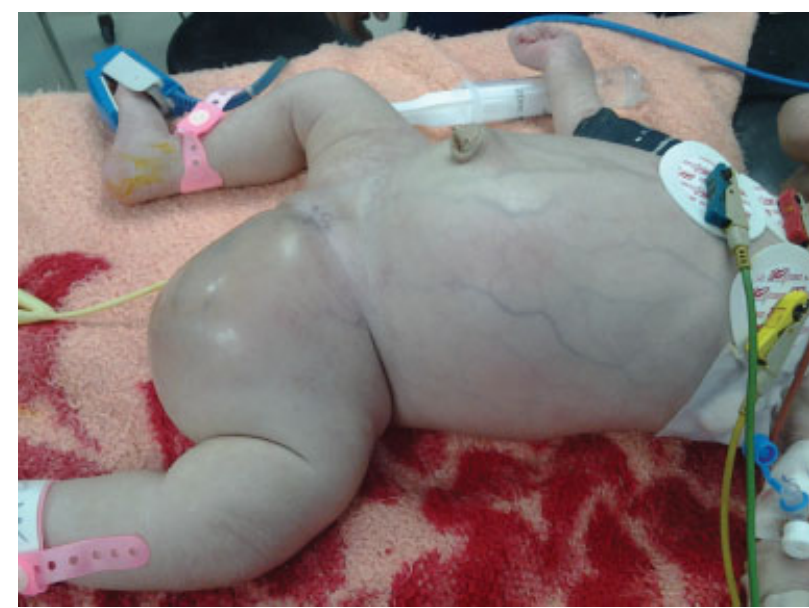

Fig.2 Preoperative clinical assessment of the patient.
The abnormal behavior related to the extension into the thigh in our case suggests that the tumor probably originated from the iliopsoas muscle, given its pelvic origin and lower limb insertion with passage under inguinal ligament. This behavior in neonates is rarely noted in literature, although a similar case was operated upon for a primary paratesticular tumor in a 13-year-old boy. ${ }^{4}$ Approximately, half of neonatal RMS arise in the bladder, vagina, testicular, and sacrococcygeal regions. ${ }^{5}$ In a multi-institutional Children's Cancer Group (CCG) study reported in 1995, a common characteristic of neonatal RMS was its aggressive biological behavior as half of the patients had widespread disease at the time of diagnosis. ${ }^{6}$

\section{Pathology}

The predominant histologic subtype in RMS presenting in neonates is embryonal subtype. These lesions are associated with allelic loss of the $11 \mathrm{p} 15$ region, ${ }^{7}$ and also coinciding with our established pathology.

\section{Management}

Surgical debulking remains our mainstay of treatment of malignant neoplasms unlike lymphatic malformations which are scheduled for watchful follow-up clinically and radiologically and are excised if complicated, especially in neonates. In this case, the progression from debulking to complete resection was attempted after careful identification and sparing of neurovascular structures followed by complete excision of both masses. However, the treatment of RMS is multidisciplinary, involving surgery, chemotherapy, and radiotherapy 
coordination. This coincides with various reviews that advocate surgery as definitive treatment modality in most neonates with solid tumors taking into account the physiologic and metabolic needs of the neonate. ${ }^{8}$

Following resection, the most effective chemotherapy regimen is considered to be vincristine, actinomycin $\mathrm{D}$, and cyclophosphamide. ${ }^{9,10}$ Complete resection of nonmetastatic primaries is recommended if it can be accomplished with acceptable morbidity. Radiotherapy is reserved for infants with gross or microscopic residual disease. ${ }^{10}$

\section{Prognosis}

Prognosis of RMS in infants younger than 1 year appears to be comparable with that of older children. ${ }^{1}$ A study compared prognosis of tumors diagnosed in the first 28 days of life with those diagnosed after 28 days. The prognosis was worse in infants diagnosed in the first 28 days of life. ${ }^{11}$ The late presentation to our hospital was attributed to the late diagnosis of the abdominal part.

Prognosis depends on stage at presentation, histologic characteristics of the lesion, and the location of the primary tumor. Infants with embryonal histology and complete surgical resection do well, with cure rates higher than $90 \%{ }^{9}$

In a study with a median follow-up of 76 months, local recurrence was the major reason for treatment failure. In particular, the local recurrence rate was high in patients who warranted radiotherapy but received none due to their age. Completeness of surgery and nodal involvement were the most significant prognostic factors. ${ }^{12}$

\section{Conclusion}

Complex neonatal malignancies require multidisciplinary management between surgery, radiology, and oncology to tailor the ideal management of each lesion. More data are needed to setup a protocol for the management of RMS.

\section{Conflict of Interest}

None.

\section{References}

1 Lobe TE, Wiener ES, Hays DM, et al. Neonatal Rhabdomyosarcoma: The IRS Experience. J Pediatr Surg 1994;29(8):1167-1170. doi:10.1016/0022-3468(94)90302-6

2 Xue H, Horwitz JR, Smith MB, et al. Malignant solid tumors in neonates: a 40-year review. J Pediatr Surg 1995;30(4):543-545

3 Moore SW, Kaschula ROC, Albertyn R, et al. The outcome of solid tumours occurring in the neonatal period. Pediatr Surg Int 1995; 10:366-370

4 Aquino MR, Gibson DP, Bloom DA. Paratesticular rhabdomyosarcoma with metastatic encasement of the abdominal aorta. Pediatr Radiol 2011;41(8):1061-1064

5 Raney RB Jr, Hays DM, Tefft M, et al. Rhabdomyosarcoma and the undifferentiated sarcomas. In: Pizzo PA, Poplack DG, eds. Principles and Pactice of Pediatric Oncology. Philadelphia, PA: Lippincott; 1989:635-658

6 Dillon PW, Whalen TV, Azizkhan RG, et al; Children's Cancer Group Surgical Committee. Neonatal soft tissue sarcomas: the influence of pathology on treatment and survival. J Pediatr Surg 1995;30(7): 1038-1041

7 Barr FG. Molecular genetics and pathogenesis of rhabdomyosarcoma. J Pediatr Hematol Oncol 1997;19(6):483-491

8 Richard G. Azizkhan, Perinatal tumors. In: Carachi R, Grosfeld JL, Azmy AF, eds. The Surgery of Childhood Tumors. 2nd ed. Berlin, Heidelberg: Springer-Verlag; 2008:146

9 Crist W, Gehan EA, Ragab AH, et al. The Third Intergroup Rhabdomyosarcoma Study. J Clin Oncol 1995;13(3):610-630

10 Crist WM, Anderson JR, Meza JL, et al. Intergroup rhabdomyosarcoma study-IV: results for patients with nonmetastatic disease. J Clin Oncol 2001;19(12):3091-3102

11 Büyükpamukçu M, Varan A, Tanyel C, et al. Solid tumors in the neonatal period. Clin Pediatr (Phila) 2003;42(1):29-34

12 Ferrari A, Casanova M, Bisogno G, et al; Italian Cooperative Group. Rhabdomyosarcoma in infants younger than one year old: a report from the Italian Cooperative Group. Cancer 2003;97(10): 2597-2604 